\title{
Interleukin-8 Overexpression Is Present in Pyoderma Gangrenosum Ulcers and Leads to Ulcer Formation in Human Skin Xenografts
}

\author{
Masahiro Oka, Carola Berking, Mark Nesbit, Kapaettu Satyamoorthy, \\ Helmut Schaider, George Murphy, Masamitsu Ichihashi, Edward Sauter, and \\ Meenhard Herlyn
}

The Wistar Institute (MO, CB, MN, KS, HS, ES, MH), Philadelphia, Pennsylvania; Thomas Jefferson University (GM, ES), Philadelphia, Pennsylvania; and Kobe University School of Medicine (MI), Kobe, Japan

SUMMARY: Interleukin-8 (IL-8) is a potent chemotactic polypeptide for neutrophils. However, the role of this cytokine during inflammation remains unclear. Skin specimens from patients with pyoderma gangrenosum demonstrated IL-8 overexpression in skin ulcers, which suggests a role for IL- 8 in the development of the disease. We therefore constructed a recombinant adenovirus expressing the complementary deoxyribonucleic acid encoding human IL-8 (IL-8/Ad5) that induces a 2000 -fold increase in IL-8 expression of infected human fibroblasts in vitro. Human skin engrafted to severe combined immunodeficiency mice and then injected with the recombinant virus demonstrated erythema, an intense perivascular infiltration of neutrophils, and extravasation of erythrocytes after 8 hours. By 12 hours after injection, neutrophils had accumulated beneath the epidermis, which then necrotized, and one or more ulcers that remained for approximately 2 weeks were observed. Clinically and histologically, the ulcers resembled pyoderma gangrenosum. These clinical and experimental findings suggest an etiologic role of IL-8 in the pathogenesis of pyoderma gangrenosum. (Lab Invest 2000, 80:595-604).

$I$ nterleukin-8 (IL-8), a member of the family of proinflammatory chemotactic cytokines with molecular masses ranging from 6 to $14 \mathrm{kd}$ (Oppenheim et al, 1991; Baggiolini et al, 1994), is a potent chemotactic factor for neutrophils. Under physiologic conditions, production of $\mathrm{IL}-8$ is not constitutive but is inducible in a variety of cell types by proinflammatory cytokines such as interleukin-1 (IL-1) and tumor necrosis factor alpha (TNF- $\alpha$ ) (Oppenheim et al, 1991; Baggiolini et al, 1994). IL-8 exerts its effects by binding to two types of receptors, CXCR1 and CXCR2 (Murphy, 1994; Baggiolini et al, 1997). These receptors are widely distributed and are found on cutaneous melanocytes, as well as on other cells (Norgauer et al, 1996). This suggests that IL-8 may possess functions other than chemoattraction. IL-8 can stimulate angiogenesis (Norrby, 1997; Kumar et al, 1998), although it appears to be less potent than classic angiogenic growth factors such as basic fibroblast growth factor and vascular endothelial growth factor and apparently acts through indirect mechanisms.

IL-8 is not detectable in normal human skin with the exception of the eccrine duct epithelium and acrosyringium (Gearing et al, 1990; Gillitzer et al, 1991; Nickoloff et al, 1991; Schulz et al, 1993). In contrast, high levels of IL-8 have been detected during acute

Received December 28, 1999.

Address reprint requests to: Dr. M. Herlyn, The Wistar Institute, 3601 Spruce Street, Philadelphia, Pennsylvania 19104. Fax: 215-898-0980; E-mail: herlynm@wista.wistar.upenn.edu inflammatory reactions (Garner et al, 1994; Ono et al, 1995; Strickland et al, 1997; Zweiman et al, 1997); in chronic skin diseases such as psoriasis vulgaris (Gearing et al, 1990; Nickoloff et al, 1991; Anttila et al, 1992; Schröder et al, 1992; Schulz et al, 1993; Gillitzer et al, 1991; Gillitzer et al, 1996), palmoplantar pustulosis (Anttila et al, 1992), and bullous pemphigoid (Schmidt, 1996); and in nonmelanoma and melanoma skin tumors (Hansen et al, 1991; Ciotti et al, 1995; Bornscheuer et al, 1996). However, the role of IL-8 in the pathogenesis of skin diseases has been difficult to elucidate because of the abundance of other cytokines that can induce IL-8 expression. For example, in T-cell lymphoma (Hansen et al, 1991) IL-8, TNF- $\alpha$, and IL-1 are all highly expressed, and in psoriasis vulgaris both IL- 8 and TNF- $\alpha$ are upregulated (Ettehadi et al, 1994). It has been difficult to determine whether IL-8 upregulation is the cause of or a response to pathologic changes. Moreover, the specific cell type(s) involved remain unclear, because all skin cells can express IL-8 when appropriately stimulated (Bonifati et al, 1994). However, there is recent evidence that different chemokines including IL-8 regulate proinflammatory functions in a unique way. For example, IL-8 and granulocyte chemotactic protein 2 (GCP-2) but not epithelial neutrophil-activating [protein] 78 (ENA-78) alter TNF- $\alpha$-induced oxidant production, and only IL-8 pretreatment inhibits chemotactic response to a later IL-8 challenge (Williams et al, 1999).

To address the effects of IL-8 in normal skin, Leonard et al (1991) injected IL-8 protein into the skin of 
healthy human subjects and observed mild infiltration of neutrophils within 30 minutes. However, there were no macroscopic changes (Leonard et al, 1991; Kemény et al, 1994). Because the biologic half-life of injected protein is expected to be short, we developed an alternative approach that was based on the intermediate-term expression of IL-8 in human skin grafts transplanted onto severe combined immunodeficiency (SCID) mice. Full-thickness grafts were injected with a recombinant adenovirus in which the CDNA encoding human IL-8 is expressed under the control of the cytomegalovirus immediate early promoter (IL-8/Ad5). Because human IL-8 is biologically active in the mouse through its ability to bind to the murine receptors (Swensson et al, 1991), we saw a strong cellular response of infiltrating murine neutrophils, which caused dramatic clinical and pathologic changes. The ulcerative lesions resembled those of pyoderma gangrenosum. We also evaluated the expression of IL-8 in clinical specimens containing pyoderma gangrenosum. In the human skin grafting experiments, we determined whether skin lesions similar to those induced by IL-8 could be induced by either TNF- $\alpha$ or monocyte chemotactic protein-1 (MCP-1).

TNF- $\alpha$ is one of a number of chemokines including $\mathrm{IL}-1$, interleukin-12, and interferon $\gamma$ that can elicit a local Shwartzman reaction, the histopathologic hallmarks of which are microthrombi in dermal vessels and hemorrhagic skin necrosis (Brozna, 1990). MCP-1 is a potent chemoattractant factor for monocytes and macrophages (Schneider et al, 1999). Our findings suggest that IL-8 plays a critical role in the pathogenesis of neutrophil-mediated skin lesions such as pyoderma gangrenosum and that other cytokines induce lesions distinct from those induced by IL-8.

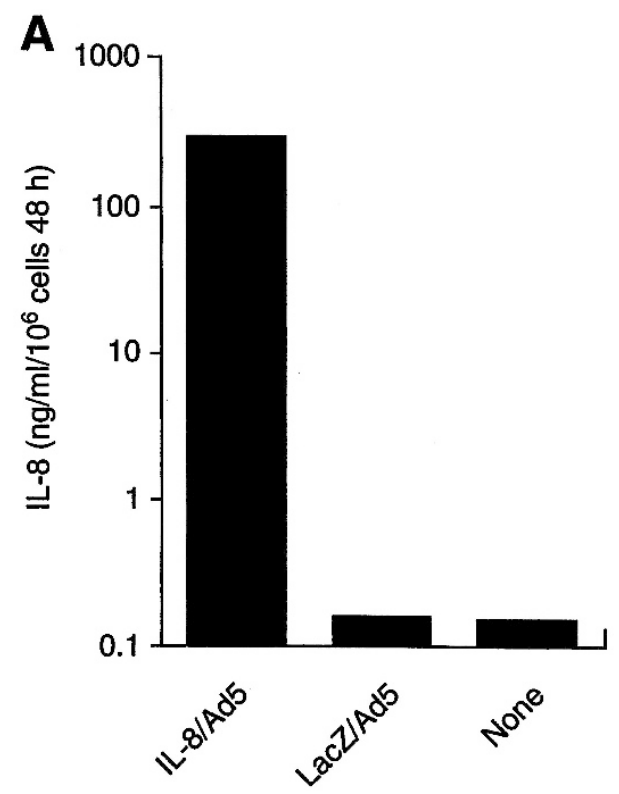

\section{Results}

\section{IL-8 Production by Human Fibroblasts Transduced with IL-8}

Twenty plaque-forming units (PFU)/cell infection of fibroblasts with IL-8/Ad5 led to a 2000-fold increase (from $0.14 \mathrm{ng} / \mathrm{ml} / 10^{6}$ cells $/ 48$ hours in control cultures to $300 \mathrm{ng} / \mathrm{ml} / 10^{6}$ cells) in IL-8 production (Fig $1 \mathrm{~A}$ ). The transduced cells expressed the specific 8-kd protein (Fig 1B). Infection of fibroblast cultures with control virus (LacZ/Ad5) had no effect on IL-8 production. There was no apparent effect on cell growth and morphologic factors after IL-8 transduction of fibroblasts. IL-8 secreted by transduced human fibroblasts was highly chemoattractive for human monocytes, as was recombinant human IL-8 (Fig 2).

\section{Ulcer Formation in Human Skin}

Human neonatal foreskins grafted onto SCID mice appeared flat, flesh-colored, and smooth (Fig. 3A). Injection of IL-8/Ad5 caused marked changes in the grafts over the 28-day observation period (Fig. 3, B to G). The earliest changes, which were evident after 8 hours (Fig. 3B), consisted of faint superficial necrosis and erythema. At 12 hours, erythema and necrosis became more severe, and numerous petechiae were visible (Fig. 3C). Small ulcers developed by 2 to 3 days (Fig. 3, D and E). The ulcers gradually enlarged, and by day 10 the lesions had developed a surface crust (Fig. 3F). Re-epithelialization that resulted in atrophic skin began after 10 to 15 days and was complete by day 28 (Fig. 3G). Injection of control virus (LacZ/Ad5) did not alter the gross appearance of the grafts at any time after injection (Fig. 3H). The adjacent mouse skin

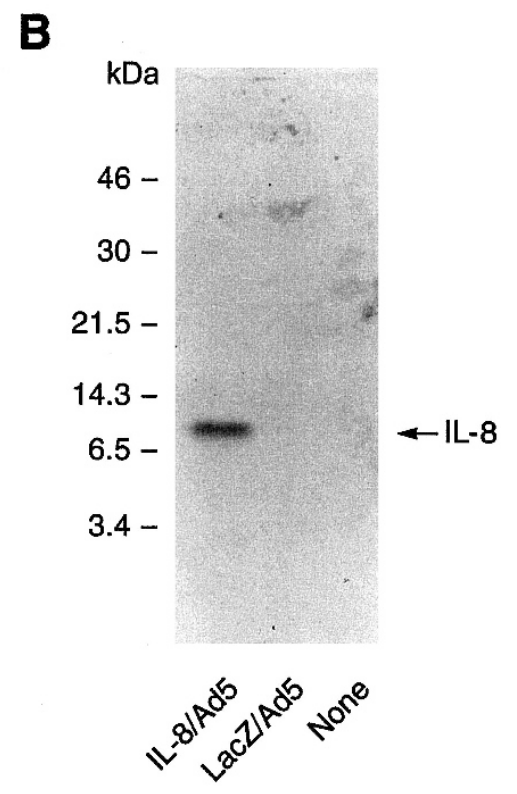

Figure 1.

Interleukin-8 (IL-8) secretion by human fibroblasts. (A) Human fibroblasts were transduced with 20 PFU/cell of IL-8/Ad5 or LacZ/Ad5 or were incubated in medium only (none) and were then cultured in serum-free medium for 48 hours, when supernatants were collected for determination of IL-8 protein content by ELISA. (B) Supernatants $(50 \mu \mathrm{l}$ ) from IL-8/Ad5- or LacZ/Ad5-transduced cells and untreated cells were subjected to Western blot analysis with anti-IL-8 mAb. The arrow indicates the migration of IL-8. 


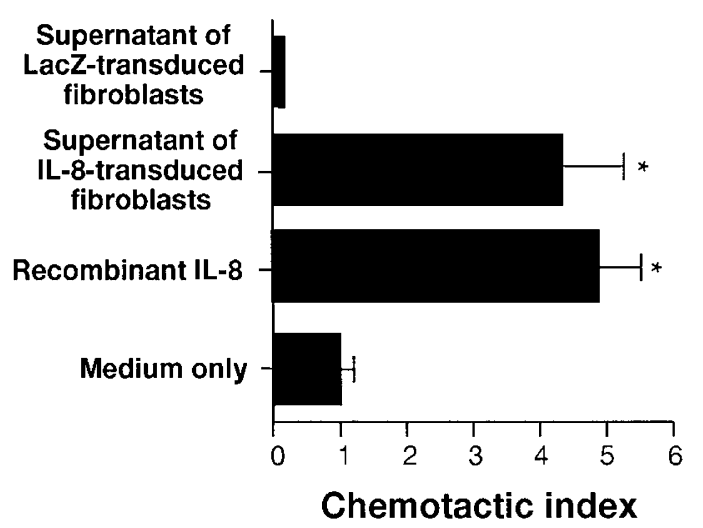

Figure 2.

Biologic activity of IL-8/Ad5-induced protein. Human fibroblasts were infected with $20 \mathrm{PFU} /$ cell of IL-8/Ad5 or LacZ/Ad5 and were cultured in serum-free medium for 48 hours, after which the supernatants were harvested. After IL-8 had been purified from the supernatants by a heparin column, biologic activity was examined in a human neutrophil chemotaxis assay that used a multiwell Boyden chamber with recombinant human IL-8 and medium alone as controls. Data, which are expressed as a chemotactic index, are the mean \pm SD of triplicate determinations. ${ }^{\star} p<0.05$ versus medium only.

remained unchanged throughout the experiment. Histologically, the epidermis was necrotic. Eight hours after IL-8 transduction, vasculitis-like changes of the superficial dermal vessels (intravascular and perivascular infiltrates of polymorphonuclear leukocytes with leukocytoclasia and erythrocyte extravasation) were noted. Numerous neutrophils infiltrated both the dermis and the epidermis. By 48 hours after transduction, complete ulceration was present, and the underlying dermis contained an intense, diffuse infiltrate (Fig. 4A) composed almost entirely of neutrophils and their fragments. Microvascular thrombi were not seen. After 28 days, when the ulcers were re-epithelialized, scar tissue had developed and the neutrophils were no longer present. In contrast, grafts transduced with LacZ at 48 hours showed only mild epidermal hyperplasia without a neutrophilic infiltrate (Fig. 4G). Epidermal changes disappeared after 10 to 15 days, and the skin became indistinguishable from noninjected skin.

The clinical diagnosis of pyoderma gangrenosum is based on skin ulcer formation (Weiss, 1989). Histologically, patient lesions show ulceration of the epidermis with a strong infiltrate of inflammatory cells (Fig. 4B). The histologic pattern of ulceration in patients' lesions is similar to that seen in skin grafts transduced with IL-8. Staining for IL-8 of both transduced grafts and patients' lesions revealed strong positivity (Fig. 4, C to F). The IL-8-transduced human skin grafts showed intense staining of fibroblasts (Fig. 4, C and E). In contrast, LacZ-transduced human skin grafts showed few to no positively stained cells (Fig. 4H), nor were sections positive that had been incubated with an unrelated control antibody (not shown). In all five cases tested, areas of acute and chronic inflammation within patients' lesions showed intense IL-8 expression, predominantly in neutrophils (Fig. 4, D and F). Skin from healthy individuals showed minimal staining with the anti-IL-8 monoclonal antibody (mAb), and sections of lesions from pyoderma gangrenosum did not stain when an unrelated mAb was used as a control (not shown).

\section{Human Skin After Injection of TNF- $\alpha$ and MCP-1/Adenovirus 5 (Ad5)}

Twenty-four hours after human neonatal foreskins grafted to SCID mice were injected with low, medium, or high-dose $(0.1,1.0$, or $10 \mu \mathrm{g})$ TNF- $\alpha$, microvascular thrombosis and erythrocyte extravasation (Fig. 5A) as well as mixed inflammatory cell infiltrates were noted in the dermis. By 48 hours, hemorrhagic skin necrosis was seen in the medium-dose group, and by day 3 there was evidence of epidermal necrosis and microscopic but not gross ulceration in all groups (not shown). Mice were killed on days 1, 2, and 3 or were not killed to evaluate the long-term effects of TNF- $\alpha$. A mouse that had received $10 \mu \mathrm{g}$ of TNF- $\alpha$ was killed on day 12 because of cachexia and apathy, which suggest a possible systemic TNF- $\alpha$ effect at this dose. A mouse that had received $0.1 \mu \mathrm{g}$ of TNF- $\alpha$ was killed on day 20 because of skin necrosis and bleeding.

In another study that compared the effects of cytokines on the development of skin lesions, we injected an adenoviral vector containing MCP-1 (MCP-1/Ad5) into human skin xenografts (unpublished data). No gross changes were observed. Histologically, a brisk mononuclear cell infiltrate was seen in the dermis and epidermis (Fig. 5B).

\section{Discussion}

Replication-deficient adenovirus is a powerful tool for human skin gene transfer because all cell types can be infected with high efficiency (Satyamoorthy et al, 1996). Infection of skin fibroblasts with our IL-8/Ad5 recombinant induced a 2000-fold increase in IL-8 secretion in vitro, and the protein was biologically active. Intradermal injection of IL-8/Ad5 into fullthickness human skin grafted to SCID mice attracted murine neutrophils, which had a rapid and dramatic effect on the skin. Within 8 hours, erythema with necrosis that became more severe during the next several hours developed on the skin surface until an ulcer formed after 48 to 72 hours. The site of IL- 8 overexpression was characterized by edema and erythrocyte extravasation accompanied by a massive infiltration of neutrophils at the intradermal injection site. The infiltration began in small superficial dermal vessels and in perivascular areas and then spread throughout the dermis and into the epidermis. The ulceration in the skin resembled pyoderma gangrenosum.

The cause of pyoderma gangrenosum is not known, but gross and microscopic evidence of acute and chronic inflammation suggest the recruitment of leukocytes through the influence of one or more cytokines. IL- 8 recruits both neutrophils and monocytes (Marie et al, 1999) and has been associated with a variety of acute inflammatory reactions (Garner et al, 1994; Ono et al, 1995; Strickland et al, 1997; Zweiman et al, 1997) and chronic skin diseases (Gearing et al, 

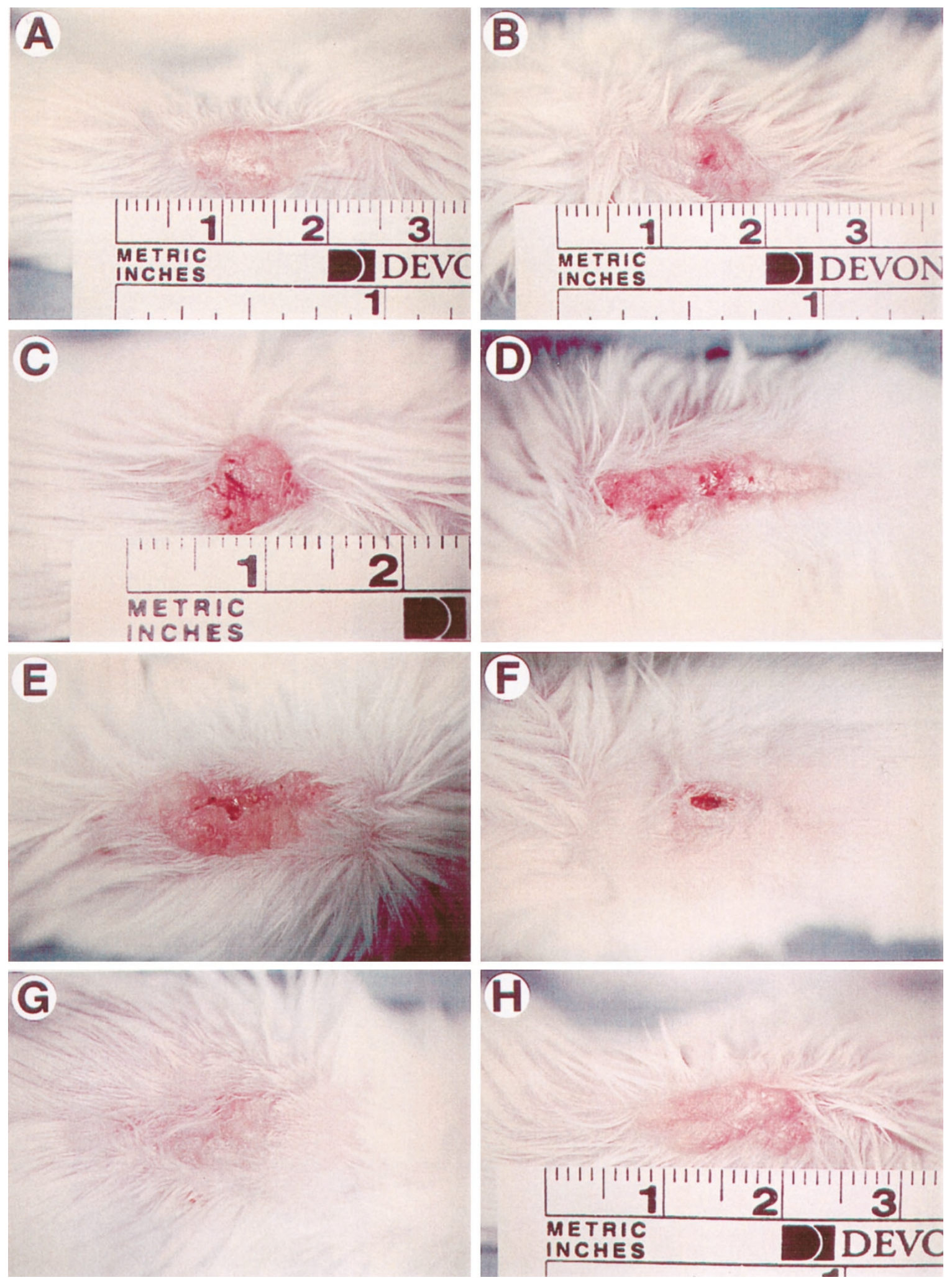

\section{Figure 3.}

Ulcer formation in human skin xenografts after IL-8/Ad5 or LacZ/Ad5 transduction. One hundred $\mu$ l of IL-8/Ad5 or LacZ/Ad5 (5 $\times 10^{8}$ PFU) was injected into human skin xenografts. Human skin (A) before transduction and after injection of IL-8/Ad5, (B) at 8 hours, (C) at 12 hours, (D) at 2 days, (E) at 3 days, (F) at 10 days, (G) at 28 days, and $(\mathrm{H}) 2$ days after injection of LacZ/Ad5. 
A
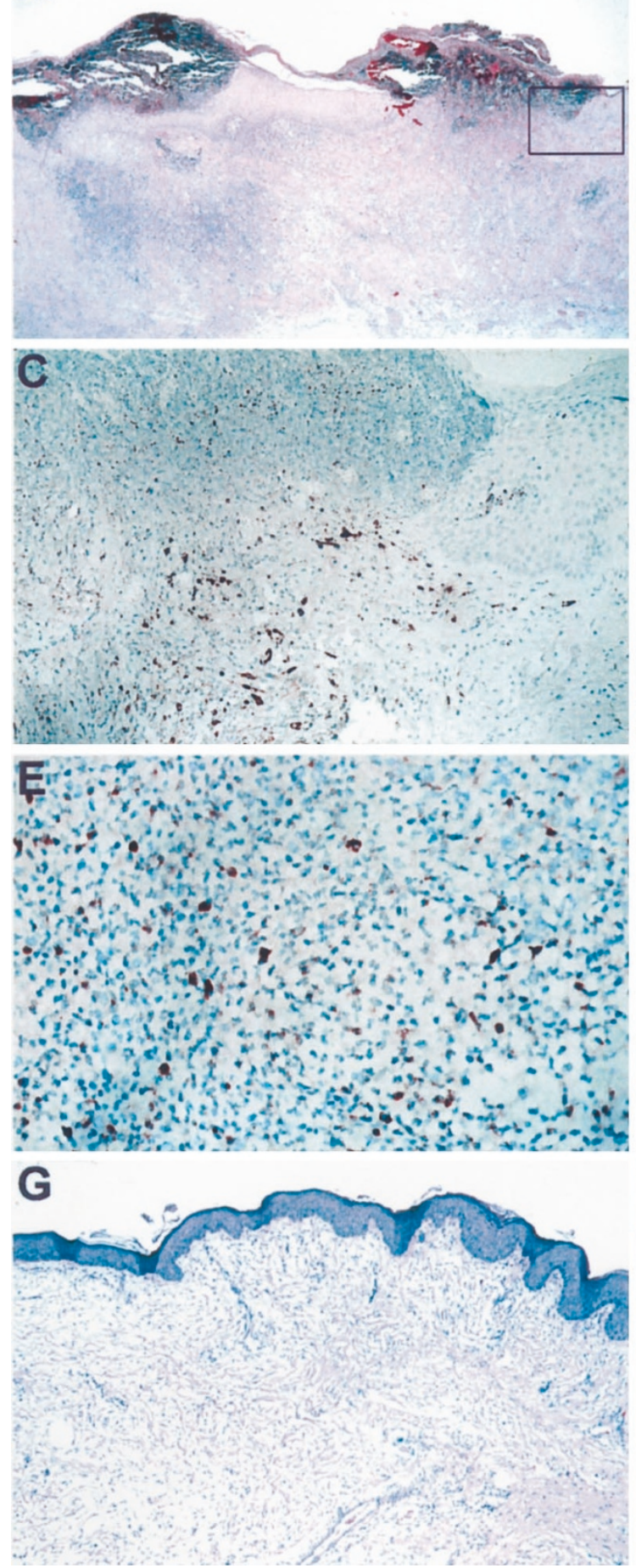

B
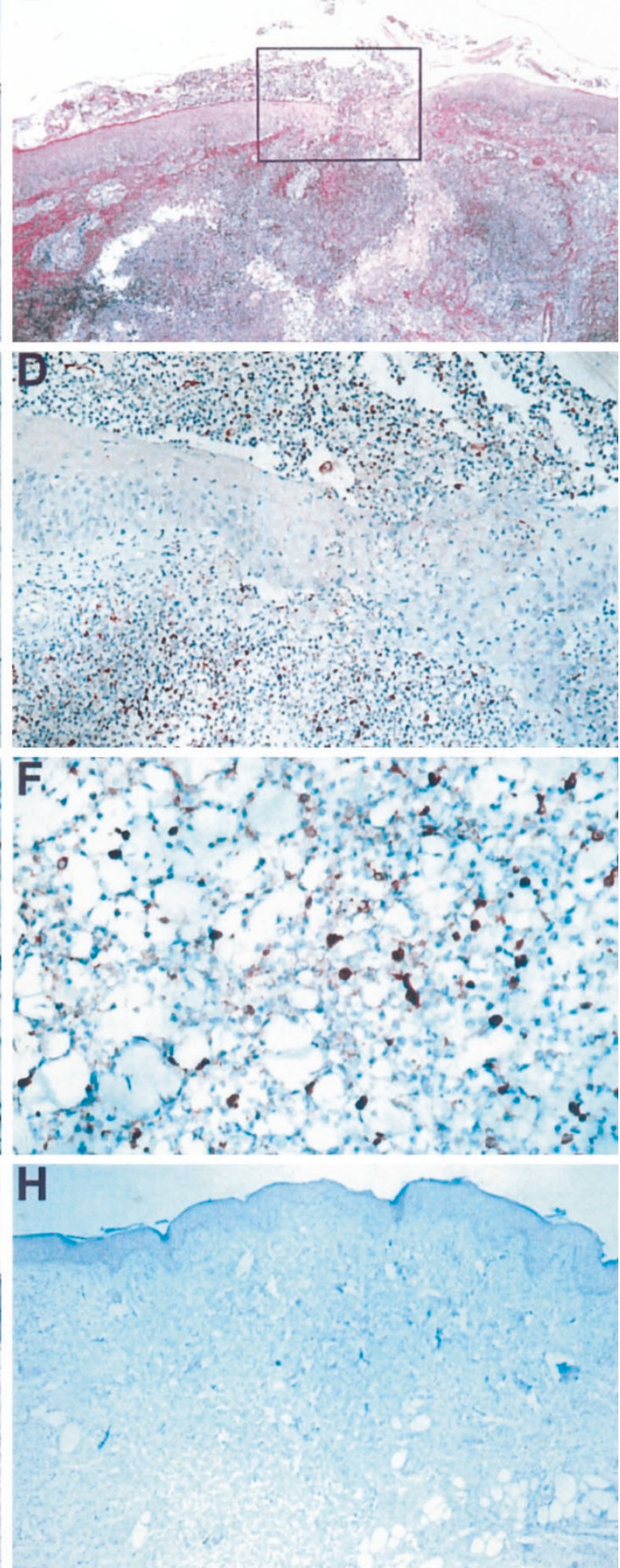

\section{Figure 4.}

Histologic changes in human skin ulcers after experimental induction with IL-8 and in clinical specimens with pyoderma gangrenosum. Histologic characteristics of skin ulcers and immunohistologic staining for IL-8 in human skin from either xenografts $(A, C, E, G, H)$ or pyoderma gangrenosum patients (B, D, F). (A) Hematoxylin and eosin $(\mathrm{H} \& \mathrm{E})$ staining of epidermal ulceration in human skin grafts 48 hours after IL-8/Ad5 infection. The dermis reveals an intense diffuse infiltrate of predominantly neutrophils $(\times 5)$. (B) Human skin from a patient with pyoderma gangrenosum; note necrosis of the epidermis with ulceration and infiltration of neutrophils $(\times 5)$. (C) Staining of a section of the xenograft from the boxed-in area in $(A)$ with anti-IL-8 mAb ( $\times 20)$. (D) Staining with anti-IL-8 mAb of the clinical specimen from the boxed-in area in $(B)(\times 20)$. (E) Staining with anti-IL-8 mAb identifies the fibroblasts of the section from $(A)(\times 40)$. $(F)$ Staining with anti-IL-8 $m A b$ identifies fibroblasts of the section from $(B)(\times 40)$. $(G)$ Human xenograft treated with LacZ and stained with $\mathrm{H} \& \mathrm{E}$. The epidermis demonstrates only mild hyperplasia without a neutrophilic infiltrate. $(\mathrm{H})$ Human xenograft treated with LacZ and stained with IL-8 monoclonal antibody. Minimal staining is observed. 


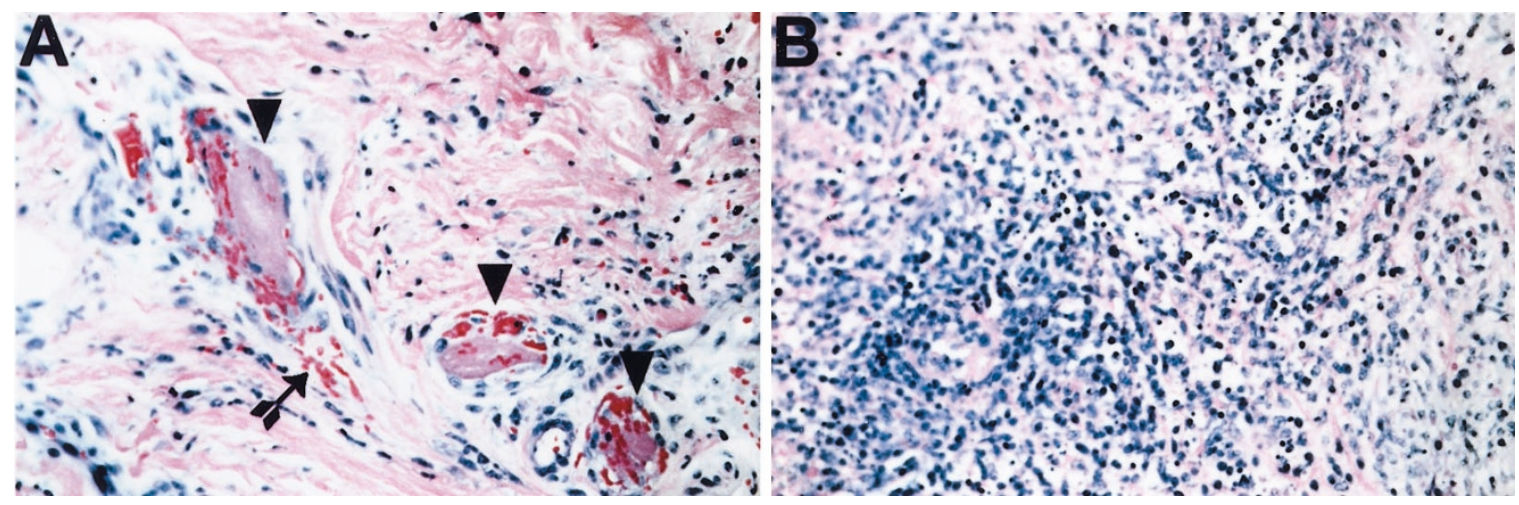

Figure 5 .

Histologic changes in human skin ulcers after experimental induction with TNF- $\alpha$ (A) and MCP-1/Ad5 (B). (A) H \& E staining $(\times 40)$ of a human skin xenograft 24 hours after the injection of $0.1 \mu \mathrm{g} \mathrm{TNF}-\alpha$. The dermis demonstrates thrombus formation (arrowheads) and erythrocyte extravasation (arrow). (B) Human skin xenograft 72 hours after injection with MCP-1/Ad5 $(\times 50)$. The dermis demonstrates a substantial mononuclear infiltrate.

1990; Nickoloff et al, 1991; Anttila et al, 1992; Schröder et al, 1992; Schulz et al, 1993; Gillitzer et al, 1991; Gillitzer et al, 1996; Schmidt, 1996), though not with pyoderma gangrenosum (Marie et al, 1999). We observed overexpression of IL-8 in areas of inflammation, with expression primarily in neutrophils in clinical specimens with pyoderma gangrenosum and in fibroblasts in experimental specimens that showed histologic changes reminiscent of pyoderma gangrenosum. Because the IL-8 staining was very intense and obscured nuclear detail, we cannot exclude the possibility that neutrophils were also stained in the experimental specimens.

Previous studies have shown that injection of natural or recombinant IL-8 into the skin of volunteers resulted in neutrophil attraction, but the neutrophils were confined to the perivascular areas with no apparent leukocytoclasis (Leonard et al, 1991; Kemény et al, 1994). Only mild (rather than gross) histologic reactions occurred, apparently because of the relatively small quantities of biologically active IL-8 that were introduced. The biologic half-life of the injected protein was estimated at several hours (Leonard et al, 1991; Swensson et al, 1991), whereas fibroblasts infected in vivo with IL-8/Ad5 expressed the transgene for 10 to 14 days (data not shown). Because IL-8 is not a mitogen for fibroblasts, resting cells may express the transgene even longer, as indicated by the persistence of ulcers for 2 weeks. Immunohistologic analyses indicate that dermal fibroblasts appear to be the main recipient cell type for the transgene signal after intradermal injection of an adenoviral vector, whereas epidermal keratinocytes showed little expression of IL-8. It remains unclear whether endothelial cells were also transduced by intradermally injected IL-8/Ad5. IL-8 molecules injected intradermally are apparently transported to postcapillary venules and small veins, which might drive leukocyte emigration from blood (Fantone and Ward, 1985; Middleton et al, 1997) or promote leukocyte-endothelial cell adhesion (Henson and Johnston, 1987). IL-8 might also be internalized by venular endothelial cells abluminally and transcytosed to the luminal surface, where it is presented to leukocytes for adherence (Middleton et al, 1997). In clinical and xenograft specimens, fibroblasts stained positive for IL-8.

Neutrophil-mediated vessel and tissue injury leading to ulcer formation involves complex mechanisms (Fantone and Ward, 1985; Henson and Johnston, 1987; Weiss, 1989; Ward and Varani, 1990; Furie and Randolph, 1995). The role of IL-8 might be not only to attract neutrophils but also to activate them to produce reactive oxygen intermediates and exocytose degradative enzymes in tissue. Activation of neutrophils can also be induced by proinflammatory cytokines such as TNF- $\alpha$ or IL- 1 that are released at the site of massive infiltration of the inflammatory cells (Weiss, 1989). Clearly, the observed leukocytoclasis is a result of neutrophil activation. The extravasation of red blood cells also suggests neutrophil-mediated damage.

We compared the effect of IL-8 on human skin xenografts with two other potent chemokines, TNF- $\alpha$ and MCP-1, to determine whether the effect observed was specific to IL-8. Chemokines (chemotactic cytokines) are the stimuli that largely control leukocyte migration. They are secreted by a variety of cells including leukocytes, epithelial cells, endothelial cells, fibroblasts, and numerous other cell types and are produced in response to both exogenous stimuli, such as viruses and bacterial LPS, and endogenous stimuli, such as IL-1, TNF, and interferon. Chemokines mediate chemotaxis and leukocyte activation. They also regulate leukocyte extravasation from the blood and/or lymph vessel luminal surface to the tissue space, the site of inflammation (Mahalingam and Karupiah, 1999). We first evaluated TNF- $\alpha$, a chemokine known to induce a local Shwartzman reaction. TNF- $\alpha$ induced intense microvascular thrombosis and erythrocyte extravasation with a relatively mild mixed inflammatory infiltrate. IL-8, however, did not induce microvascular thrombosis but rather an intense inflammatory infiltrate of predominantly polymorphonuclear leukocytes in the dermis and epidermis. The third cytokine tested, MCP-1, induced a diffuse mononu- 
clear cell infiltrate with only scattered neutrophils that did not lead to necrotic lesions in the skin.

Intense infiltration of neutrophils characterizes several diseases such as pyoderma gangrenosum, Sweet's syndrome, rheumatoid neutrophilic dermatosis, and bowel-associated dermatosis syndrome (Weiss, 1989). The clinical and pathologic pattern seen in our study most closely resembles that of pyoderma gangrenosum, a rare ulcerating skin disease of unknown cause (Schwaegerle et al, 1988; Weiss, 1989). Typically, pyoderma gangrenosum begins as small erythematous papules that spread concentrically. The central portion of the lesions rapidly undergoes necrosis and ulceration and finally heals with atrophic scarring. Lesions of pyoderma gangrenosum exhibit necrosis and ulceration of the epidermis and dermis, as well as a heavy infiltration of neutrophils at the margins of the lesions and chronic inflammatory cells at the base. Epidermal acanthosis is seen in areas immediately adjacent to the ulcer. The degree of vessel involvement ranges from none to fibrinoid necrosis. In most biopsied lesions, a neutrophilic infiltrate with limited vascular damage is present. Thus IL-8 overexpression may be involved in the pathogenesis of this disease. Pyoderma gangrenosum is often associated with ulcerative colitis (Mahida et al, 1992; Nielsen et al, 1997), Crohn's disease (lzzo et al, 1993), and rheumatoid arthritis (Feldmann et al, 1996), which suggests that IL-8 overexpression plays a role in a variety of inflammatory diseases. Although IL-8 appears to cause neutrophil infiltration in these inflammatory diseases, our study could not address the underlying mechanism of IL-8 activation in tissue. Controlling the activators of $\mathrm{IL}-8$ or inactivating this chemokine may lead to effective reduction of neutrophil-induced tissue damage.

\section{Materials and Methods}

\section{Cells and Cell Culture}

Normal human fibroblasts obtained from culture explants of newborn foreskin were cultured in Dulbecco's modified Eagle's medium (DMEM) (GIBCO/BRL, Grand Island, New York) supplemented with 10\% fetal calf serum (FCS) (Irvine Scientific, Irvine, California). The cells were used between passages 3 and 10 . Human embryonic 293 kidney cells were maintained in DMEM supplemented with $10 \%$ FCS.

\section{Generation of Recombinant Adenovirus Carrying the Human IL-8 Gene}

To construct the IL-8/Ad5 recombinant, 5' and 3' NotI primers containing an EcoRl site were used to generate a cDNA by polymerase chain reaction amplification in which the full-length IL-8 cDNA (Rot, 1991; Matsushima et al, 1988) was used as the template. The resulting DNA was subcloned into pUCBM20 (Boehringer Mannheim, Indianapolis, Indiana), was sequenced to exclude mutations, and was subcloned into the Notl site of the adenoviral vector pAdCMV (obtained from the Vector Core, The Wistar Institute,
Philadelphia, Pennsylvania). DNA of dl7001/Ad5 lacking the E1 and E3 genes (Ranheim et al, 1993) was cut with Clal, after which it was calcium phosphateprecipitated with Nhel-digested pAdCMV containing the IL-8 gene in sense orientation and was then incubated with human embryonic 293 cells containing the E1 gene of Ad5. Cells were incubated in serumfree medium for 2 hours, after which serum was added to a final concentration of $10 \%$. After 5 hours, 293 cells were treated with a $10 \%$ glycerol shock for 2 minutes in serum-free DMEM. Cells were then grown overnight in DMEM containing 10\% FCS, and an overlay containing $0.8 \%$ agar (DIFCO, Detroit, Michigan) in Eagle basal medium (GIBCO/BRL) supplemented with $2 \% \mathrm{FCS}$ and 0.5 mmole $\mathrm{MgCl}_{2}$ was applied. Fresh overlay was added every 4 days until individual plaques appeared, typically by day 16 . Plaques were expanded in 293 cells grown in 35- $\mathrm{mm}$ dishes (Falcon; Becton Dickinson Labware, Lincoln Park, New Jersey), and fresh medium was added every other day until cytopathic effects were noted. Cells were then harvested, and adenoviral deoxyribonucleic acid (DNA) was isolated and cut with BamHI before electrophoresis in a 1\% agarose gel. Southern blot analysis using the Notl/BamHI fragment of the IL-8 gene as the template for random priming was performed to select positive plaques. After two additional rounds of plaque purification and Southern blot analysis, a single plaque was selected to obtain a large preparation of recombinant adenovirus. IL-8/Ad5 was purified by cesium chloride gradient centrifugation, and the final PFU were determined by titration on 293 cells under an agar overlay. LacZ/Ad5, an E1/E3 defective recombinant virus producing $\beta$-galactosidase, was prepared by the Vector Core (The Wistar Institute) for use in control studies.

\section{Generation of Recombinant Adenovirus Carrying the MCP-1 Gene}

A plasmid containing the 741 base pair human MCP-1 cDNA was used in producing the adenoviral vector MCP-1/Ad5. The MCP-1 cDNA encompassing the open reading frame of $\mathrm{MCP}-1$ ( $\sim 400$ base pair) was subcloned into a modified pSL301 vector (Vector Core) by EcoRI and Pst digestion of both MCP-1 cDNA and the pSL301 vector. pSL301 containing the MCP-1 cDNA was subcloned into the Notl site of the adenoviral vector pAdCMV (Vector Core). dl7001/Ad5 DNA was cut with Clal, was calcium phosphateprecipitated with Nhel-digested pAdCMV containing the MCP-1 gene in sense orientation, and was incubated with human embryonic 293 cells. The remainder of the preparation was as described above for IL-8.

\section{IL-8 Immunoassays}

Human fibroblasts were infected with $20 \mathrm{PFU} /$ cell of either IL-8/Ad5 or LacZ/Ad5 and were cultured in serum-free AIM V medium (GIBCO/BRL) for 48 hours. IL-8 protein content in the supernatants was measured in triplicate with an ELISA kit (Biosource Inter- 
national, Camarillo, California) according to the manufacturer's instruction. For Western blot analysis, 50 $\mu$ of the supernatant was heated to $100^{\circ} \mathrm{C}$ for 10 minutes in an equivalent amount of SDS sample buffer (10\% SDS, 100 mmole Tris [pH 6.8], 1\% glycerol, 125 $\mathrm{mg} / \mathrm{ml}$ bromophenol blue, 5\% [volume per volume ] 2-mercaptoethanol [12.5 M]), and was separated on a $15 \%$ SDS-polyacrylamide gel. The gel was electroblotted to polyvinylidene difluoride membranes (Millipore Corp., Bedford, Massachusetts) overnight at $40 \mathrm{v}$. Membranes were washed with $2 \%$ bovine serum albumin in phosphate-buffered saline (PBS) containing $0.05 \%$ Tween 20 , were then incubated for 2 hours at room temperature with murine antihuman IL-8 monoclonal antibodies (Quantikine; RD-Systems, Minneapolis, Minnesota), and were further incubated for 2 hours at room temperature with goat antimouse immunoglobulin G labeled with alkaline phosphatase (Jackson Immuno Research Laboratories Inc., West Grove, Pennsylvania). Immunoreactive bands were visualized by incubating membranes with 5-bromo-4chloro-3-indolyl phosphate and nitroblue tetrazolium (Sigma, St. Louis, Missouri).

\section{Preparation of Neutrophils and Chemotaxis Assays}

Human neutrophils were isolated from the peripheral blood of healthy donors by gradient centrifugation on Ficoll-Hypaque (Pharmacia, Piscataway, New Jersey) as described (Wei et al, 1993). When this isolation technique is used, the purity and viability of neutrophils are more than 98\% (Wei et al, 1993). The biologic activity of IL- 8 in the culture supernatants of human fibroblasts was examined by a neutrophil chemotaxis assay. IL-8 protein was purified by adsorption and elution from a heparin column, and chemotaxis was evaluated in multiwell Boyden chambers (Neuroprobe, Cabin John, Maryland) by filling bottom wells with bovine serum albumin (control), recombinant human IL-8, or test samples and by adding neutrophils resuspended in bovine serum albumin-containing medium to the upper wells. After 50 minutes' incubation at $37^{\circ} \mathrm{C}$, filters ( $3 \mu \mathrm{m}$ pore size) were removed, were wiped on the upper side, and were stained with Diff-Quik 1 and 2. Neutrophils on the lower side of the filter were counted in 4-10 $\times 10$ power fields for each well. A chemotactic index defined as the cell number in the test sample/cell number in the control group was calculated. Each sample was examined in triplicate.

\section{SCID Mouse Engraftment and Injection}

C.B.-17 SCID mice (Bosma and Caroll, 1991) were bred and maintained in The Wistar Institute Animal Facility. Human neonatal foreskins obtained from area hospitals were used for grafting on the dorsum of anesthetized SCID mice within 48 hours after removal (Soballe et al, 1996). Four to 6 weeks later, after healing had occurred, the foreskin xenografts were injected with a single dose of one of the following: $5 \times$ $10^{8}$ PFU of IL-8/Ad5 in $100 \mu$ l culture medium; LacZ/
Ad5 in $100 \mu$ l culture medium (both 10 mice/group); $0.1,1$, and $10 \mu \mathrm{g}$ recombinant human TNF- $\alpha$ in $100 \mu \mathrm{l}$ PBS (University of Pennsylvania, Philadelphia, Pennsylvania) (4 mice/group); or $5 \times 10^{8} \mathrm{PFU}$ of MCP-1/ Ad5 in $50 \mu \mathrm{l}$ PBS (4 mice/group). Mice from each group were then killed, and histologic and immunohistochemical analyses of the grafts were performed.

\section{Histologic Factors and Immunohistochemistry}

Histologic Factors. Xenografts were dissected free from surrounding murine tissue, were fixed overnight in $10 \%$ neutral-buffered formalin, and were paraffinembedded. Replicate serial sections from each graft were stained with hematoxylin and eosin (H \& E). Five clinical specimens from subjects with both gross and microscopic evidence of pyoderma gangrenosum were obtained from the Department of Pathology, Kobe University School of Medicine (Kobe, Japan) and were fixed and stained in a fashion similar to that of the xenograft skin specimens.

Immunohistochemistry. Five-micrometer tissue sections were incubated with either an antihuman IL-8 monoclonal antibody (R\&D, Minneapolis, Minnesota) or an isotype-matched control antibody (Dako Corp., Carpinteria, California) overnight at $4^{\circ} \mathrm{C}$ and were washed in PBS followed by a three-step immunoperoxidase method using an avidin-biotin horseradish peroxidase (Vector Laboratories, Burlingame, California). Immunoreactivity was visualized by means of 3,3'diaminobenzidine (Sigma, St. Louis, Missouri) with a counterstain of Gill's hematoxylin No. 1 (Fisher Scientific, Malvern, Pennsylvania).

\section{Acknowledgements}

The authors thank all members of the Wistar Institute editorial department for their excellent assistance. This work was supported by grants CA-47159, CA25874, and CA-10815.

\section{References}

Anttila HSI, Reitamo S, Erkko P, Ceska M, Moser B, and Babbiolini M (1992). Interleukin-8 immunoreactivity in the skin of healthy subjects and patients with palmoplantar pustulosis and psoriasis. J Invest Dermatol 98:96-101.

Baggiolini M, Dewald B, and Moser B (1994). Interleukin-8 and related chemotactic cytokines-CXC and CC chemokines. Adv Immunol 55:97-197.

Baggiolini M, Dewald B, and Moser B (1997). Human chemokines: An update. Annu Rev Immunol 15:675-705.

Bonifati C, Carducci M, Cordiali FP, Trento E, Sacerdoti G, Fazio M, and Ameglio F (1994). Correlated increases of tumour necrosis factor- $\alpha$, interleukin- 6 and granulocyte monocyte-colony stimulating factor levels in suction blister fluids and sera of psoriatic patients - Relationships with disease severity. Clin Exp Dermatol 19:383-387.

Bornscheuer E, Schröder JM, Christophers E, and Sticherling M (1996). Interleukin-8 immunoreactivity in malignant tumours of the skin. Acta Derm Venereol 76:210-213. 
Bosma MJ and Caroll AM (1991). The SCID mouse mutant: Definition, characterization, and potential uses. Annu Rev Immunol 9:323-350.

Brozna JP (1990). Shwartzman reaction. Semin Thromb Hemost 16:326-332.

Ciotti $P$, Rainero ML, Nicolò G, Spina B, Garrè C, Casabona F, Santi PL, and Bianchi-Scarrà G (1995). Cytokine expression in human primary and metastatic melanoma cells: Analysis in fresh bioptic specimens. Melanoma Res 5:41-47.

Ettehadi P, Greaves MW, Wallach D, Aderka D, and Camp RDR (1994).z Elevated tumour necrosis factor-alpha (TNF- $\alpha$ ) biological activity in psoriatic skin lesions. Clin Exp Immunol 96:146-151.

Fantone JC and Ward PA (1985). Polymorphonuclear leukocyte-mediated cell and tissue injury: Oxygen metabolites and their relations to human disease. Hum Pathol 16:973-978.

Feldmann M, Brennan FM, and Maini RN (1996). Role of cytokines in rheumatoid arthritis. Annu Rev Immunol 14:397440.

Furie MB and Randolph GJ (1995). Chemokines and tissue injury. Am J Pathol 146:1287-1301.

Garner WL, Rodriguez JL, Miller CG, Till GO, Rees RS, Smith DJ, and Remick DG (1994). Acute skin injury releases neutrophil chemoattractants. Surgery 116:42-48.

Gearing AJH, Fincham NJ, Bird CR, Wadhwa M, Meager A, Cartwright JE, and Camp RDR (1990). Cytokines in skin lesions of psoriasis. Cytokine 2:68-75.

Gillitzer R, Berger R, Mielke V, Müller C, Wolff K, and Stingl G (1991). Upper keratinocytes of psoriatic skin lesions express high levels of NAP-1/IL-8 mRNA in situ. J Invest Dermatol 97:73-79.

Gillitzer R, Ritter U, Spandau U, Goebeler M, and Bröcker EB (1996). Differential expression of GRO- $\alpha$ and IL-8 mRNA in psoriasis: A model for neutrophil migration and accumulation in vivo. J Invest Dermatol 107:778-782.

Hansen ER, Vejlsgaard GL, Lisby S, Heidenheim M, and Baadsgaard O (1991). Epidermal interleukin $1 \alpha$ functional activity and interleukin-8 immunoreactivity are increased in patients with cutaneous T-cell lymphoma. J Invest Dermatol 97:818-823.

Henson PM and Johnston RB Jr (1987). Tissue injury in inflammation, oxidants, proteinases, and cationicproteins. $\mathrm{J}$ Clin Invest 79:669-674.

Izzo RS, Witkon K, Chen Al, Hadjiyane C, Weinstein MI, and Pellecchia C (1993). Neutrophil-activating peptide (interleukin-8) in colonic mucosa from patients with Crohn's disease. Scand J Gastroenterol 28:296-300.

Kemény L, Ruzicka T, Dobozy A, and Michel G (1994). Role of interleukin-8 receptor in skin. Int Arch Allergy Immunol 104: 317-322.

Kumar R, Yoneda J, Bucana CD, and Fidler IJ (1998). Regulation of distinct steps of angiogenesis by different angiogenic molecules. Int J Oncol 12:749-757.

Leonard EJ, Yoshimura T, Tanaka S, and Raffeld M (1991). Neutrophil recruitment by intradermally injected neutrophil attractant/activation protein-1. J Invest Dermatol 96:690694.
Mahalingam S and Karupiah G (1999). Chemokines and chemokine receptors in infectious diseases. Immunol Cell Biol 77:469-475.

Mahida YR, Ceska M, Effenberger F, Kurlak L, Lindley I, and Hawkey CJ (1992). Enhanced synthesis of neutrophilactivating peptide-1/interleukin-8 in active ulcerative colitis. Clin Sci (Colch) 82:273-275.

Marie C, Roman-Roman S, and Rawadi G (1999). Involvement of mitogen-activated protein kinase pathways in interleukin-8 production by human monocytes and polymorphonuclear cells stimulated with lipopolysaccharide or $\mathrm{My}$ coplasma fermentans membrane lipoproteins. Infect Immun 67:688-693.

Matsushima K, Morishita K, Yoshimura T, Lavu S, Kobayashi Y, Lew W, Appella W, Kung HF, Lepnard EJ, and Oppenheim JJ (1988). Molecular cloning of a human monocyte-derived neutrophil chemotactic factor (MDNCF) and the induction of MDNCF mRNA by interleukin 1 and tumor necrosis factor. $J$ Exp Med 167:1883-1893.

Middleton J, Neil S, Wintle J, Clark-Lewis I, Moore H, Lam C, Auer M, Hub E, and Rot A (1997). Transcytosis and surface presentation of IL-8 by venular endothelial cells. Cell 91:385395.

Murphy PH (1994). The molecular biology of leukocyte chemoattractant receptors. Annu Rev Immunol 12:593-633.

Nickoloff BJ, Karabin GD, Barker JNWN, Griffiths CEM, Sarma V, Mitra RS, Elder JT, Kunkel SL, and Dixit VM (1991). Cellular localization of interleukin-8 and its inducer, tumor necrosis factor-alpha in psoriasis. Am J Pathol 138:129-140.

Nielsen OH, Rüdiger N, Gaustanes M, and Horn T (1997). Intestinal interleukin-8 concentration and gene expression in inflammatory bowel disease. Scand J Gastroenterol 32: 1028-1034.

Norgauer J, Metzner B, and Schraufstatter I (1996). Expression and growth-promoting function of the IL-8 receptor beta in human melanoma cells. J Immunol 156:1132-1137.

Norrby K (1997). Mast cells and de novo angiogenesis: Angiogenic capability of individual mast-cell mediators such as histamine, TNF, IL-8, and bFGF. Inflamm Res 46:7-8.

Ono I, Gunji H, Zhang JZ, Maruyama K, and Kaneko F (1995). A study of cytokines in burn blister fluid related to wound healing. Burns 21:352-355.

Oppenheim JJ, Zachariae COC, Mukaida N, and Matsushima K (1991). Properties of the novel proinflammatory supergene "intercrine" cytokine family. Annu Rev Immunol 9:617-648.

Ranheim TS, Shisler J, Horton TM, Wold LJ, Gooding LR, and Wold WS (1993). Characterization of mutants within the gene for the adenovirus E3 14.7-kilodalton protein which prevents cytolysis by tumor necrosis factor. J Virol 67:2159-2167.

Rot A (1991). Chemotactic potency of recombinant human neutrophil attractant/activation protein-1 (interleukin-8) for polymorphonuclear leukocytes of different species. Cytokine 3:21-27.

Satyamoorthy K, Nesbit M, Hsu M-Y, and Herlyn M (1996). Utility of adenoviruses as gene expression modules in melanoma. In: Maio M, editor. Immunology of human melanoma. Amsterdam: IOS Press, 71-77.

Schmidt E (1996). Elevated levels of interleukin-8 in blister fluid of bullous pemphigoid compared with suction blisters of healthy control subjects. J Am Acad Dermatol 34:310-312. 
Schneider A, Panzer U, Zahner G, Wenzel U, Wolf G, Thaiss F, Helmchen U, and Stahl RA (1999). Monocyte chemoattractant protein-1 mediates collagen deposition in experimental glomerulonephritis by transforming growth factor-beta. Kidney Int 56:135-144.

Schröder JM, Gregory H, Young J, and Christophers E (1992). Neutrophil-activating proteins in psoriasis. J Invest Dermatol 98:241-247.

Schulz BS, Michel G, Wagner S, Süss R, Beetz A, Peter RU, Kemény L, and Ruzicka T (1993). Increased expression of epidermal IL-8 receptor in psoriasis. Down-regulation by FK-506 in vitro. J Immunol 151:4399-4406.

Schwaegerle SM, Bergfeld WF, Sentzer D, and Tidrick RT (1988). Pyoderma gangrenosum: A review. J Am Acad Dermatol 18:559-568.

Soballe PW, Montone KT, Satyammorthy K, Nesbit M, and Herlyn M (1996). Carcinogenesis in human skin grafted to SCID mice. Cancer Res 56:757-764.

Strickland I, Rhodes LE, Flanagan BF, and Friedmann PS (1997). TNF- $\alpha$ and IL-8 are upregulated in the epidermis of normal human skin after UVB exposure: Correlation with neutrophil accumulation and E-selectin expression. J Invest Dermatol 108:763-768.
Swensson O, Schubert C, Christophers E, and Schröder J-M (1991). Inflammatory properties of neutrophil-activating protein-1/interleukin 8 (NAP-1/L-8) in human skin: A light- and electron microscopic study. J Invest Dermatol 96:682-689.

Ward PA and Varani J (1990). Mechanisms of neutrophilmediated killing of endothelial cells. J Leukoc Biol 48:97-102.

Wei S, Blanchard DK, Liu JH, Leonard WJ, and Djeu JY (1993). Activation of tumor necrosis factor- $\alpha$ production from human neutrophils by IL-2 via IL-2-R $\beta$. J Immunol 150:19791987.

Weiss SJ (1989). Tissue destruction by neutrophils. N Engl J Med 320:365-376.

Williams MA, Cave CM, Quaid G, and Solomkin JS (1999). Chemokine regulation of neutrophil function in surgical inflammation. Arch Surg 134:1360-1366.

Zweiman B, Kaplan AP, Tong L, and Moskovitz AR (1997). Cytokine levels and inflammatory responses in developing late-phase allergic reactions in the skin. J Allergy Clin Immunol 100:104-109. 